\title{
Orthogonal projection-based harmonic signal removal for operational modal analysis
}

\author{
S. Gres ${ }^{1}$, P. Andersen ${ }^{2}$, C. Hoen ${ }^{3}$, and L. Damkilde ${ }^{1}$ \\ ${ }^{1}$ Department of Civil and Structural Engineering, Aalborg University, Thomas Manns Vej 23, Aalborg, DK 9000, \\ 2 Structural Vibration Solutions A/S, NOVI Science Park, Niels Jernes Vej 10, Aalborg, DK 9220, \\ ${ }^{3}$ Kongsberg Digital AS, Drengsrudbekken 12, Asker, NOR 1383,
}

\begin{abstract}
A presence of a high amplitude periodic signals in the output responses from operating structures often pose a challenge for output-only system identification and, in case of health monitoring, damage detection/localization methods. This paper introduces a pre-processing approach that removes the harmonic part from the output signals directly in the time domain. The new method uses orthogonal projections of the harmonic realization of the signal onto the raw time series within the stochastic subspace framework. Proposed algorithm is tested on two experimental examples. First, an aluminum plate excited with both random white and periodic excitations. Second, a full-scale industrial case of a ferry excited by a random environmental load with harmonic interference from a rotating machinery on-board. In both cases the proposed method removes the harmonics from the structural responses while leaving the random part of the output signal.
\end{abstract}

Keywords: Operational Modal Analysis, Stochastic Subspace Identification, Rotating Machinery, Harmonic Removal, Nonstationary Harmonics

\section{INTRODUCTION}

Subspace-based system identification methods became practical tools for identification of the eigenstructure of linear vibrating systems, notably for capacity of solving large models and for the consistency in parameter estimates under non-stationary noise excitations [1]. A family of output-only stochastic subspace-based algorithms (SSI) is deployed for Operational Modal Analysis (OMA) in fields such as aeronautic, mechanical and biomedical engineering, for examples see [2], and formulates basis for a number of Structural Health Monitoring (SHM) methods like in [3], [4]. However, using subspace-based algorithms for OMA and SHM is problematic when applied to structures with rotating machinery, like wind turbines, diesel engines or helicopters, due to the presence of harmonic inputs. In context of a system subjected to a random load combined with steady state signal, the subspace-based methods identify the harmonics as very lightly damped modes that one can filter in the mode selection process. Difficulties emerge when the harmonic excitations are non-stationary, coincide with the structural modes or are of high energy that masks the system response to the random part of the input. In that case, a desirable solution is to discard the harmonic influence over the output signal prior to system identification and without additional knowledge of such, e.g. tachometer measurements. A development of such harmonic removal method in SSI framework is the focus of the present paper.

In context of reducing the harmonic peaks from the response of the system prior to OMA a number of methods exists. Timesynchronous-averaging (TSA) is a signal processing technique that extracts periodic waveforms from signals by averaging synchronized blocks of the signal in the angular domain. In application to harmonic removal the averaged signal is subtracted from the raw measurements, what results in removal of the frequencies depicted to synchronize the blocks, for examples see [5]. Angle matching is often achieved via measurements of a signal with a frequency equivalent to harmonic excitation, which is not always available in some of the real-life applications and was attempted to overcome in [6]. A family of methods that does not require tachometer measurements are based on cepstrum (an inverse Fourier transform of the logarithm of the spectrum). $A$ number of applications of the cepstral lifters to harmonic removal can be found in [7]. A relatively new group of methods is based on estimating a parametric harmonic signal model fitted via e.g. numerical Gauss-Newton algorithm, see [8], or non-parametric methods, see [9], which estimate is later subtracted from the measurements. 
The method proposed in this paper computes a harmonic realization of a modal state space model that is fitted to the recorded responses and projected orthogonally onto the raw signals. The paper is organized as follows: the next section contains derivation of the method, subsequently both the experimental and full-scale results are presented in the section 3 and section 4 concludes the paper.

\section{THEORETICAL FRAMEWORK}

First paragraph of this section recalls the main principles of the SSI algorithm, in particular the concept of orthogonal projections and innovation component of the data that lead to proposed harmonic reduction method, which description follows in the next paragraph.

\subsection{State space modeling}

Consider a second order differential equation of motion (1) to model a behavior of a $d$ degree of freedom, viscously damped, linear time-invariant (LTI) structural system,

$$
\left\{\begin{array}{l}
M \ddot{u}(t)+D \dot{u}(t)+K u(t)=v(t) \\
y(t)=C_{a} \ddot{u}+C_{v} \dot{u}(t)+C_{d} u(t)
\end{array}\right.
$$

where $M, D, K \in \mathbb{R}^{d x d}$ are mass, damping and stiffness matrices respectively; $C_{a}, C_{v}, C_{d} \in \mathbb{R}^{r x d}$ are selection matrices for accelerations, velocities and displacements where $r$ is a number of sensors; $u(t), v(t) \in \mathbb{R}^{d x 1}$ denote a vector of displacements and a vector of external forces respectively; $y(t) \in \mathbb{R}^{r x 1}$ is a output vector; dot expresses a derivative with respect to time $t$. Providing that input $v(t)$ is unmeasured, transform (1) to a stochastic state-space form and sample it with $k$ discrete time steps $\Delta t$, what writes,

$$
\left\{\begin{array}{l}
x_{k+1}=A x_{k}+v_{k} \\
y_{k}=C x_{k}+w_{k}
\end{array}\right.
$$

where the system states $x_{k}=\left[u(k \Delta t)^{T} \dot{u}(k \Delta t)^{T}\right]^{T}$; zero mean white noise vectors $v_{k}$ and $w_{k}$ denote the process and output noise respectively; $A \in \mathbb{R}^{n x n}, C \in \mathbb{R}^{r x n}$ are the state and observation matrices with,

$$
A=\exp \left(\left[\begin{array}{cc}
0 & I \\
-M^{-1} K & -M^{-1} D
\end{array}\right] \Delta t\right), \quad C=\left[\begin{array}{cc}
C_{d} & 0 \\
0 & C_{v} \\
-C_{a}{ }^{-1} M^{-1} K & -C_{a}{ }^{-1} M^{-1} D
\end{array}\right]
$$

where $n$ is a model order.

While the system matrices $\{A, C\}$ and subsequently the modal parameters- natural frequencies $f$, damping ratios $\zeta$ and mode shapes $\phi$ can be identified from (2) with covariance driven SSI (SSI-cov), see [10], we pursue data driven algorithm (SSI-data), see [11], with associated construction of Kalman filter to formulate the optimal prediction of the states and the output itself.

Let $z_{k}=E\left(x_{k} \mid y_{k-1}\right)$ be the approximation of $x_{k}$ given the past data vector $y_{k-1}$ where $E\left(x_{k} \mid y_{k-1}\right)$ denotes the image of $x_{k}$ orthogonally projected onto the subspace spanned by the stacked vector of past observations $y_{k-1}$ with,

$$
E(x \mid y)=E\left(x y^{T}\right) E\left(y y^{T}\right)^{-1} y
$$

where $E(\cdot)$ denotes the expectation operator. Provided that both $x_{k}$ and $y_{k-1}$ are jointly normally distributed the expression $E\left(x_{k} \mid y_{k-1}\right)$ is the conditional expectation, see [12], and yields the best linear (least square) prediction of $x_{k}$ given $y_{k-1}$. A step forward predictor of $z_{k}$ writes $z_{k+1}=E\left(x_{k+1} \mid y_{k}\right)$ which, combined with the innovation component of the data $e_{k}=y_{k}-$ $y_{k \mid k-1, k-2, \ldots, 1}$, gives $z_{k+1}=E\left(x_{k+1} \mid y_{k-1}\right)+E\left(x_{k+1} \mid e_{k}\right)$. By definition, the residual $e_{k}$ and the past data sequence $y_{k-1, k-2, \ldots 1}$ are not correlated. Substitute $z_{k+1}$ with (2) to formulate the innovation state space model,

$$
\begin{aligned}
& z_{k+1}=A z_{k}+K e_{k} \\
& y_{k}=C z_{k}+e_{k}
\end{aligned}
$$

where $K=E\left(x_{k} e_{k}\right) E\left(e_{k} e_{k}\right)^{-1}$ is the steady state Kalman filter sequence. To predict a time response of the LTI system from the matrices $\{A, C, K\}$ given inputs $y_{k}$ insert (4b) into (4a) to obtain,

$$
\begin{aligned}
& z_{k+1}=(A-K C) z_{k}+K y_{k} \\
& \hat{y}_{k}=C z_{k}
\end{aligned}
$$

where $\hat{y}_{k}$ is the realization of the response and the innovations $e_{k}=y_{k}-\hat{y}_{k}$ are the prediction errors. 


\subsection{Harmonic reduction}

The procedure that follows consists of three steps: first a similarity transform of the innovation state space to a modal state space, second, a prediction of modes that correspond to the harmonic frequencies- selected prior to the reduction or detected with automated methods such as in [13] and last an orthogonal projection of raw time series onto the harmonic realization of the output.

A linear transformation of the Kalman states (4a) to modal coordinates, for reference see [14], is given by,

$$
\hat{z_{t}}=V z_{t}
$$

where $V$ denotes a transformation matrix and yields right eigenvectors of $A$,

$$
A V=\lambda V, \quad \lambda \in \mathbb{C}^{n x n}, \quad V \in \mathbb{C}^{r x n}
$$

Substituting the Kalman states from (4a) and (4b) with its similarity transforms, the innovation state space model takes a modal form and is represented by,

$$
\left\{\begin{array}{l}
\hat{z}_{t+1}=V^{-1} A V \hat{z_{t}}+V^{-1} K e_{t} \\
y_{t}=C V \hat{z_{t}}+e_{t}
\end{array}\right.
$$

where the transformed state matrices can be written as $A_{0}=V^{-1} A V \in \mathbb{C}^{n x n}, C_{0}=C V \in \mathbb{C}^{r x n}, K_{0}=V^{-1} K \in \mathbb{C}^{n x r}$. Notice that $A_{0}=\lambda$ and $C_{0}=\phi$, where the latter implies that a realization of the output in the modal form will render the response of the model due to identified modes. That manifests with,

$$
\begin{aligned}
& \hat{z}_{t+1}=\left(A_{0}-K_{0} C_{0}\right) \hat{z_{t}}+K_{0} y_{t} \\
& \hat{y}_{t}=C_{0} I_{m} \hat{z_{t}}
\end{aligned}
$$

where $I_{m} \in \mathbb{R}^{n x n}$ is a diagonal selection matrix with ones on the diagonal entries that corresponds to the pairs of complex conjugate eigenvalues of $A$ reflecting the response from selected modes.

Suppose that the state space system from (4a) is completely controllable and observable, that is $C V_{n} \neq 0 \forall n$ and $V_{n}^{-1} K \neq 0 \forall n$ and $i_{\text {har }}$ being a Boolean vector mapping the complex conjugate eigenvalues of $A$ to the frequency of harmonic input. To remove the harmonic modes from the system response, first consider a prediction of the harmonic part given by,

$$
\hat{y}_{h a r}=C_{0} \operatorname{diag}\left(i_{\text {har }}\right) \hat{z_{t}}
$$

Subsequently, one can decouple the true response of the system from the harmonic-affected response by projecting the latter orthogonally onto harmonic realization $\hat{y}_{h a r}$, what writes,

$$
E\left(y_{t} \mid \hat{y}_{h a r}\right)=y_{t}-E\left(y_{t} \hat{y}_{h a r}^{T}\right) E\left(\hat{y}_{h a r} \hat{y}_{h a r}^{T}\right)^{-1} \hat{y}_{h a r}
$$

where the resultant residual is orthogonal to the subspace spanned by $\hat{y}_{h a r}$ and, given a complete identification of the harmonic modes, yields a reduced matrix,

$$
\hat{y}_{\text {red }}=E\left(y_{t} \mid \hat{y}_{\text {har }}\right)
$$

with the harmonics removed.

\section{RESULTS}

The efficiency of the orthogonal projection-based harmonic removal is investigated with two laboratory tests of a plate and a full-scale, in-operation, dynamic test of a ship. The performance of the method is assessed based on its capacity to remove the harmonic poles from the measurements while maintaining the natural modal properties of the structure unaffected. A variation of the method is implemented in ARTeMIS Modal Pro 5.2, for reference see [15], which is used here for signal processing and presentation of results. 


\subsection{Experimental results}

The measurements were collected from a rectangular aluminum plate subjected to both random and harmonic excitation and equipped with a 16-channel acquisition system recording accelerations normal to the plate surface. The experimental tests were carried out by Niels-Jørgen Jacobsen from B\&K Nærum in Denmark. The plate model and the experimental setup are illustrated on Figures 1 and 2 . The signals were sampled with $4096 \mathrm{~Hz}$ over 120 s intervals.

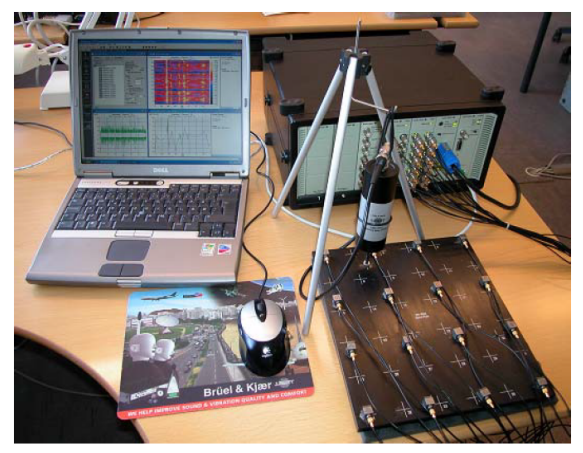

Figure 1: The experimental setup: test plate with 16 acceleration channels, shaker, acquisition system.

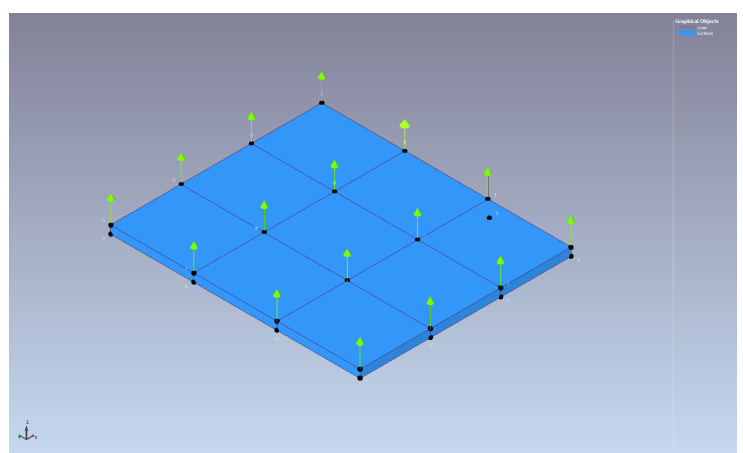

Figure 2: The test plate model with 16 acceleration channels attached.

To challenge the harmonic reduction algorithm two non-trivial input schemes are considered, namely:

- Random combined with swept sine of frequency varying in between $340-342 \mathrm{~Hz}$ and a sweep rate of $10 \mathrm{~Hz} / \mathrm{s}$,

- Random combined with burst sine of frequency of $370 \mathrm{~Hz}$ and 10 s burst intervals.

A short-time Fourier transforms (STFT) of the recorded accelerations for the two scenarios are depicted on Figures 3 and 4.
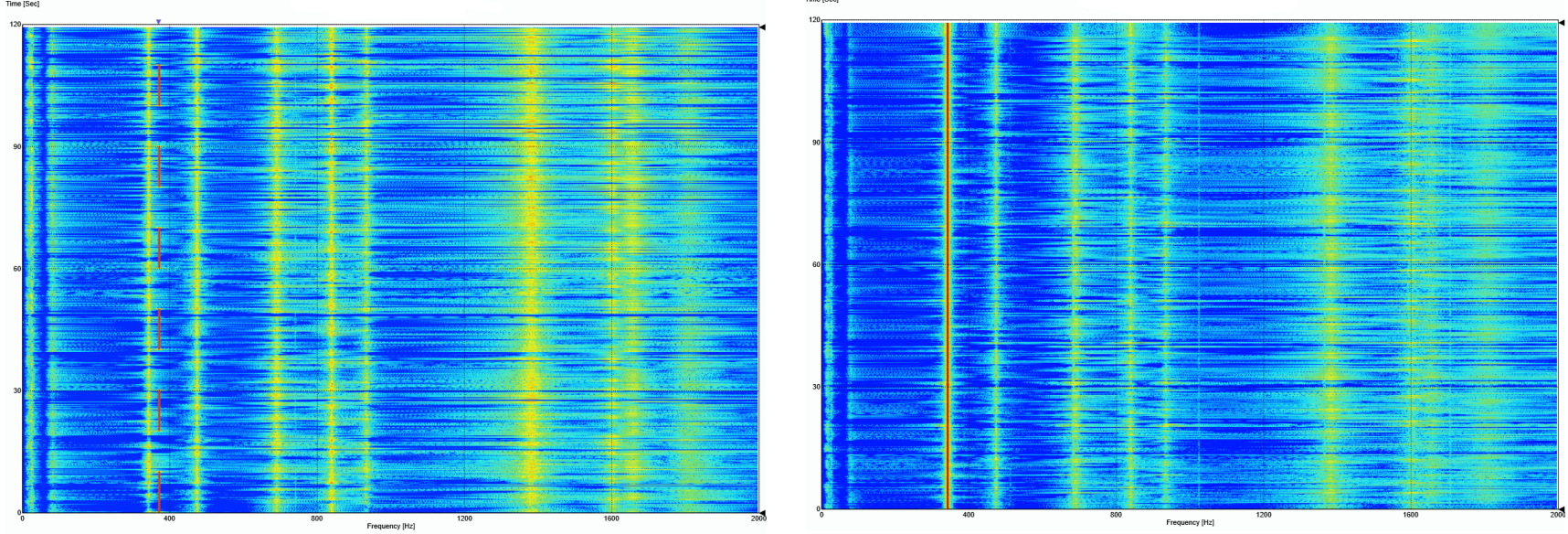

Figure 3: STFT channel 1-3 before the harmonic reduction. Figure 4: STFT channel 1-3 before the harmonic reduction. Burst sine: $370 \mathrm{~Hz}, 10 \mathrm{~s}$. 
The non-stationary harmonic frequencies in the measured accelerations are represented as a $5 \mathrm{~dB}$ dash red line in Figure 3 and $20 \mathrm{~dB}$ bold red line in Figure 4. A power difference between the steady state and the system response is $10-25 \mathrm{~dB}$, Figure 3 and Figure 4. Small fluctuations $(2 \mathrm{~Hz})$ of the swept sine will become visible after zooming in the Figure 4 . In both cases harmonics are in the vicinity, for the swept sine directly on top, of the $1^{\text {st }}$ natural frequency of the plate, where the latter is $341 \mathrm{~Hz}$, see Table 1. In context of OMA, performing the system identification on the two tested data sets even with the harmonic-robust methods is ineffective (high model orders, multiple spurious modes and unstable stabilization diagrams), hereby the reduction attempt is fully justified.

The analysis requires several user-defined parameters. First, the algorithm proposed in (9) and (10) operates in the premise that the harmonic frequencies are selected prior to the analysis and as such, we pick a sharp peak of the first singular values (SVs) of the power spectral densities (PSD) of the measured accelerations yielding the frequency of the harmonic input. Second, we define a number of block rows in the data Hankel matrix and a range of damping ratios to navigate the identification algorithm towards a state space model that predicts the harmonic modes in a optimal way. The subject of the optimum is not discussed in this work.

Both experimental tests are processed with one set of the user-defined inputs, namely: 45 block-rows of data Hankel matrix and $0.00-0.1 \%$ range of the harmonic mode damping ratios. The manually picked frequency of the harmonic response is $370 \mathrm{~Hz}$ and $341 \mathrm{~Hz}$ respectively for burst sine and swept sine. The example of a graphical user interface containing the input parameters and SVs of PSD from the data before and after the harmonic reduction, for the burst sine case, is illustrated on Figure 5.

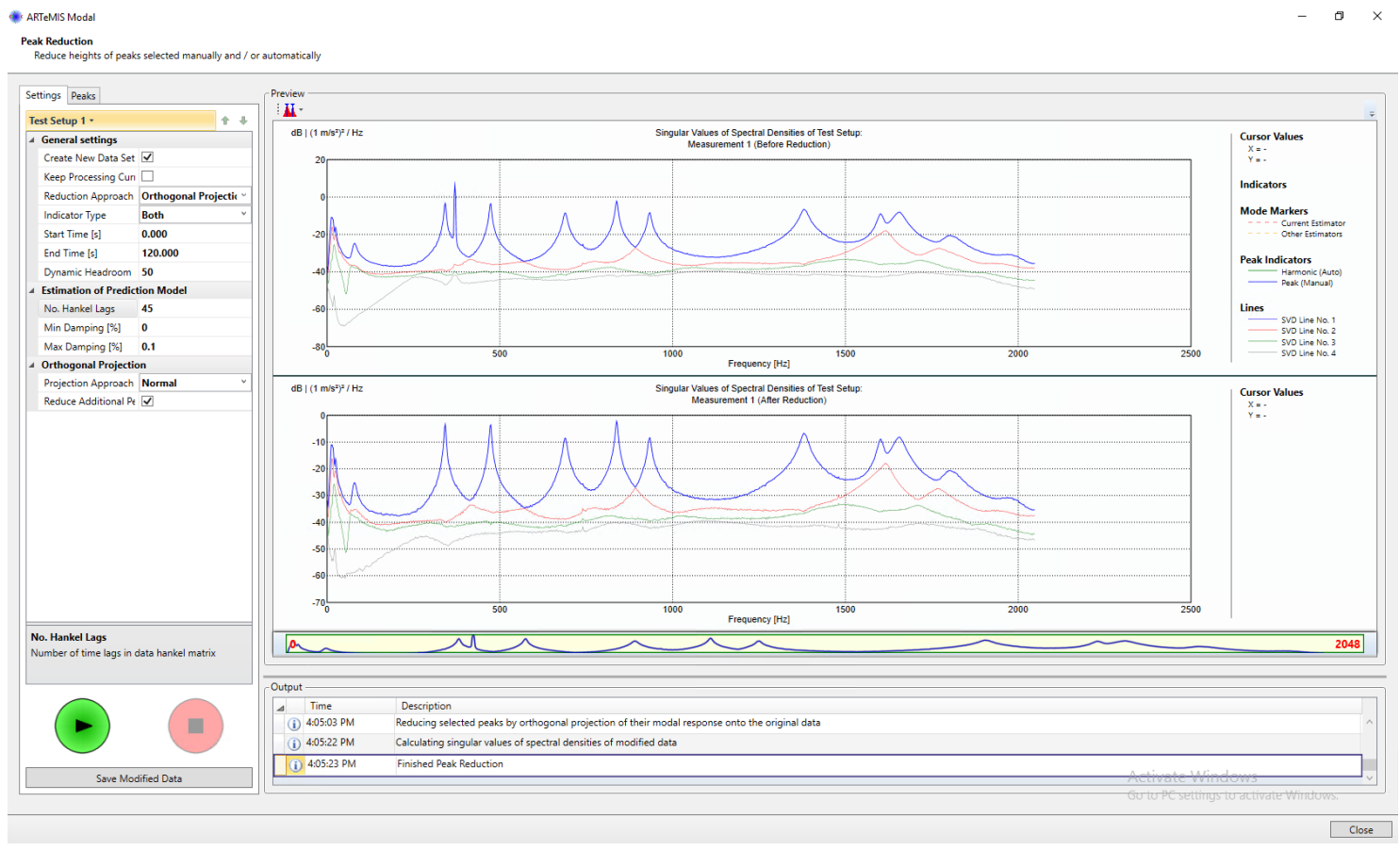

Figure 5: Removal harmonic at $370 \mathrm{~Hz}$. Burst sine: $370 \mathrm{~Hz}, 10 \mathrm{~s}$.

A visual inspection of Figure 5 shows a successful removal of harmonics at $370 \mathrm{~Hz}$, what is in agreement with the STFT of the modified data, see Figure 6, and confirmed by the comparison of the modal properties of the plate subjected to random vibrations and the OMA of the measurements after harmonic removal, see Table 1 . In case of swept sine, harmonics are removed recursively in a second trial run with the same input parameters; the results are depicted on Figure 7 and Table 1. 


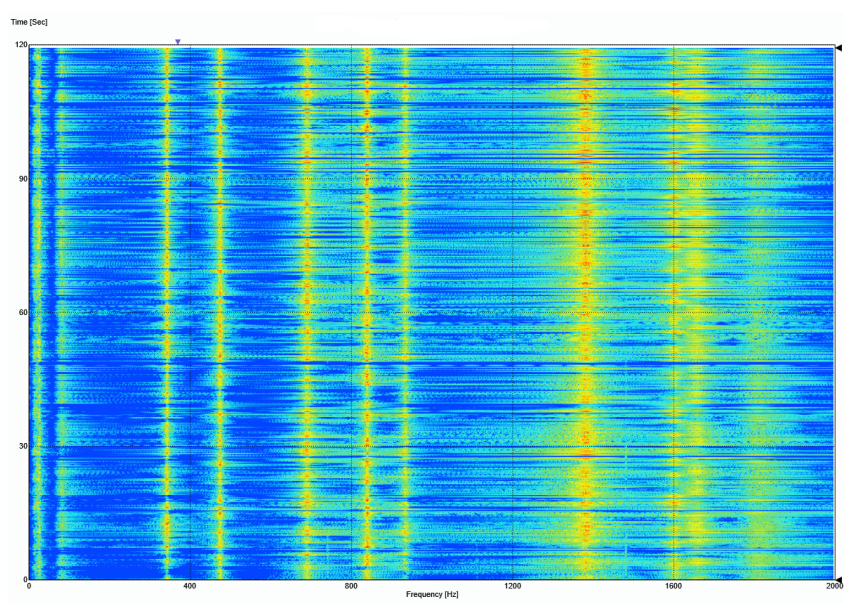

Figure 6: STFT channel 1-3 after the harmonic reduction. Burst sine: $370 \mathrm{~Hz}, 10 \mathrm{~s}$.

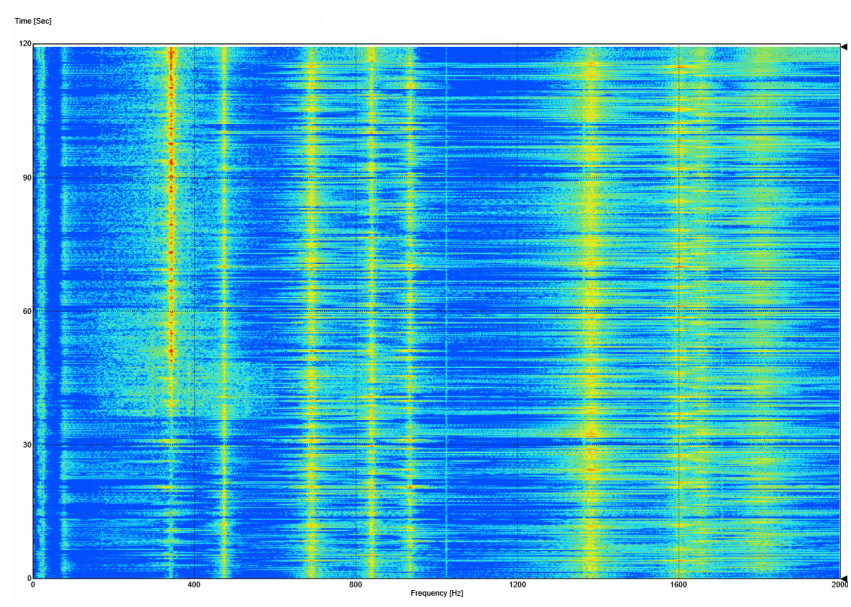

Figure 7: STFT channel 1-3 after the harmonic reduction. Swept sine: $340-342 \mathrm{~Hz}, 10 \mathrm{~Hz}$.

Figure 6 illustrates that the response of the plate to the burst sine input (dashed red line on Figure 3) is removed without leaving any additional harmonic artifacts in the modified signals. As for the swept sine test, the Figure 7 indicates that after the harmonic reduction the magnitude of the power spectrum in the $340-342 \mathrm{~Hz}$ range dropped from $20 \mathrm{~dB}$ to $6 \mathrm{~dB}$, although, since the frequency of the harmonics coincide with the $1^{\text {st }}$ natural frequency of the plate, it is not a direct sign that the harmonics are completely removed. To verify both cases, we refer to Table 1 containing an overview of the modal properties of the plate extracted both from random vibration measurements and the modified signals after the harmonic removal. The influence of the shaker weight has been included. Modal properties are estimated with an output-only SSI with Extended Unweighted Principal Component (UPCX) method, for reference see [16].

\begin{tabular}{lcccccc}
\hline Test [-] & $f_{1}[\mathrm{~Hz}]$ & $f_{2}[\mathrm{~Hz}]$ & $f_{3}[\mathrm{~Hz}]$ & $\zeta_{1}[\%]$ & $\zeta_{2}[\%]$ & $\zeta_{3}[\%]$ \\
\hline Random vibration & 341.01 & 472.10 & 688.60 & 0.56 & 0.49 & 0.88 \\
Random + swept sine & 341.33 & 472.62 & 688.50 & 0.57 & 0.45 & 0.84 \\
Random + burst sine & 341.18 & 472.89 & 688.88 & 0.59 & 0.47 & 0.81 \\
\hline
\end{tabular}

TABLE 1: Three first natural frequencies and damping ratios of the plate estimated with SSI-UPCX.

Considering that the results of the random vibrations test are the true modal parameters of the plate, the natural frequency of the first three modes exhibits a marginal deviation of max. $0.5 \%$ between the different data sets. That is not the case for the damping ratio. For the swept sine test: the smallest error yields $1.42 \%$ in the $1^{\text {st }}$ mode and the highest error yields $9.92 \%$ in the $2^{\text {nd }}$ mode. For the burst sine test: the smallest error writes $4.25 \%$ in the $2^{\text {nd }}$ mode and the highest error writes $4.97 \%$ in the $1^{\text {st }}$ mode. The highest errors are significant, in particular when recalling that e.g. the swept sine $(340-342 \mathrm{~Hz})$ input is mixed with the $1^{\text {st }}$ mode $(341.01 \mathrm{~Hz})$ instead of the $2^{\text {nd }}(472.10 \mathrm{~Hz})$, see Figure 5 . One possible reason for that bias can be a non-linear damping behavior of the plate while loaded by a high amplitude period input; other might be a non-optimal choice of the state space model to predict the harmonic response of the signal.

\subsection{Full-scale test}

The ferry is excited by a random environmental load (wind and waves) with a harmonic interference from a rotating machinery on-board. The ferry docks at Flensburg shipyard, is $199.8 \mathrm{~m}$ long and powered by $20 \mathrm{MW} 9$ cylinder MAN B\&W engine with nominal rotation speed of 123rpm. The dynamic test is undergone under fully operational conditions; the responses are recorded using a 16-channel acquisition system and sampled with $128 \mathrm{~Hz}$. The measurements were carried out by Dr. Sven-Erik Rosenow, 
Santiago Uhlenbrock and Prof. Günter Schlottmann from University of Rostock in Germany and made available to analysis by Structural Vibration Solutions from Aalborg, Denmark. The same data set was analyzed in [17] and [18] with respect to harmonic detection. Model of the ship with the measured degrees of freedom is illustrated on Figures 8 and 9 . Prior to harmonic reduction measurements are decimated to $16 \mathrm{~Hz}$.

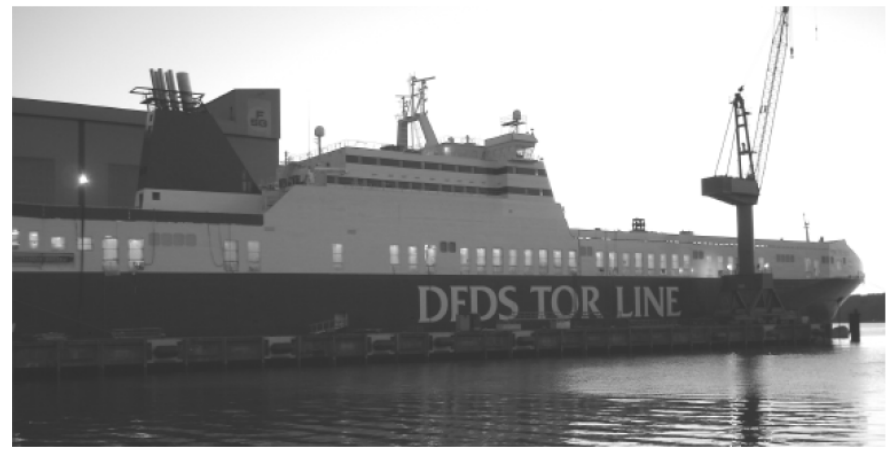

Figure 8: The ferry at the Flensburg shipyard.

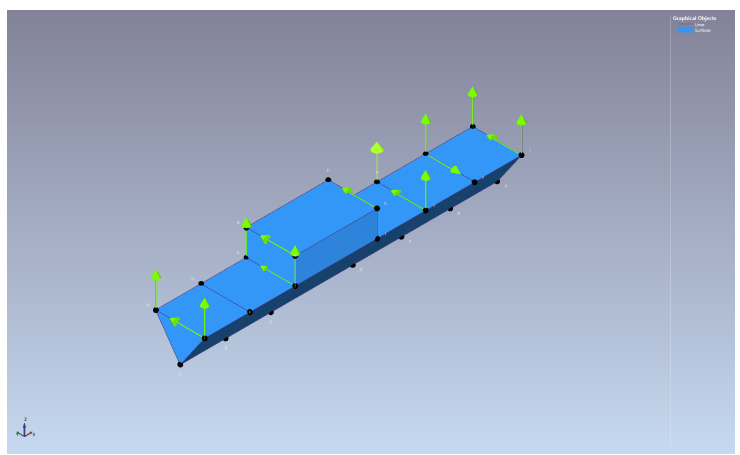

Figure 9: The ferry model with 16 acceleration channels attached.

During the test the rotational frequency of the engine is $2.05 \mathrm{~Hz}$, hence a family of four harmonic frequencies: $2.05 \mathrm{~Hz}, 4.1 \mathrm{~Hz}$, $6.15 \mathrm{~Hz}$ and $7.8 \mathrm{~Hz}$ is present in the decimated response signals, see Figure 10. The harmonic reduction is prepared with user-defined parameters depicted on Figure 10, with two harmonic peaks at $2.05 \mathrm{~Hz}$ and $4.1 \mathrm{~Hz}$ selected to reduce.

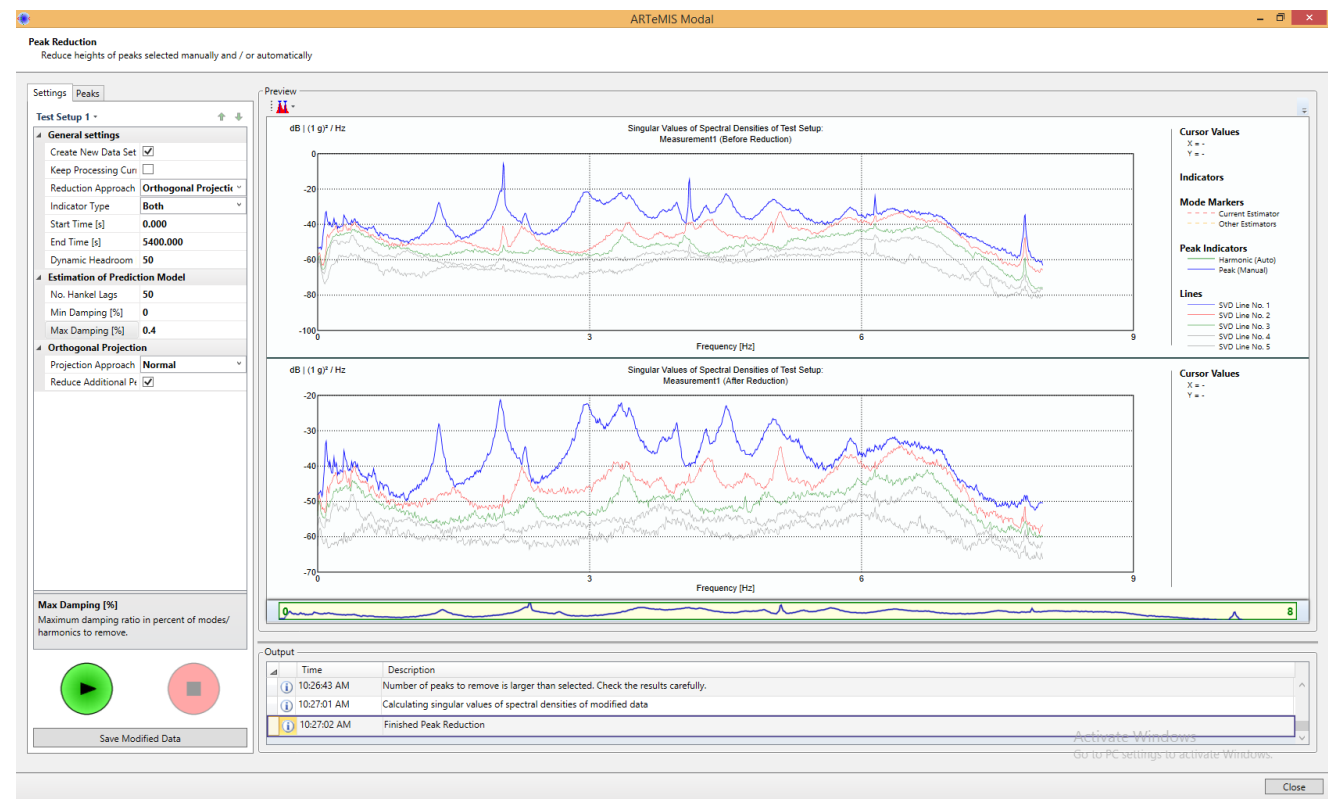

Figure 10: Removal of predefined harmonics at $2.05 \mathrm{~Hz}$ and $4.1 \mathrm{~Hz}$. Harmonics at $6.15 \mathrm{~Hz}$ and $7.8 \mathrm{~Hz}$ removed automatically.

Figure 10 illustrates that along with peaks at $2.05 \mathrm{~Hz}$ and $4.1 \mathrm{~Hz}$ the algorithm automatically reduced the harmonics at $6.15 \mathrm{~Hz}$ and $7.8 \mathrm{~Hz}$. A marginal remanence of the harmonic mode at $4.1 \mathrm{~Hz}$ is still present in the second largest SV as well as an artifact of the $6.15 \mathrm{~Hz}$ peak. That fact, however, is not expected to have influence on the system identification of the modified signals. 
For more graphic overview Figures 11 and 12 show the STFTs of the measurements before and after the harmonic reduction.

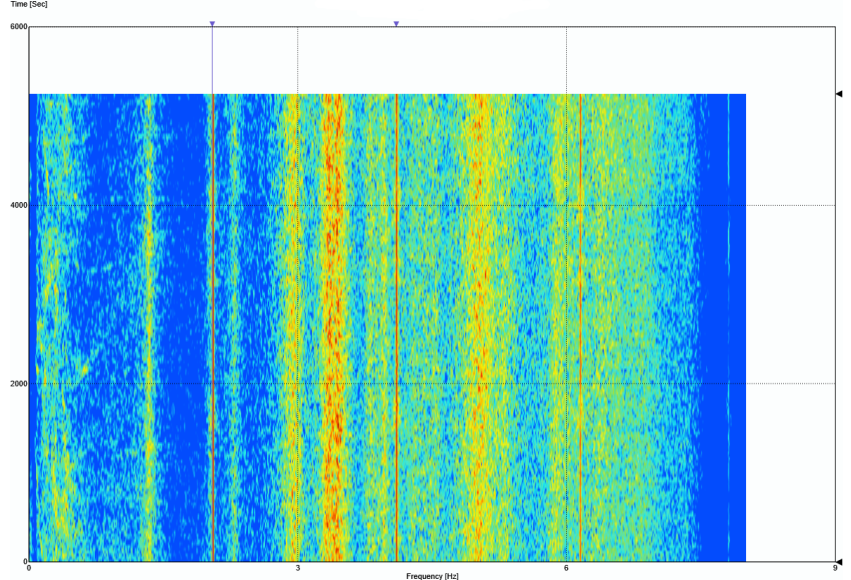

Figure 11: STFT channel 5-9 before the harmonic reduction. Harmonics at $2.05 \mathrm{~Hz}, 4.1 \mathrm{~Hz}, 6.15 \mathrm{~Hz}$ and $7.8 \mathrm{~Hz}$.

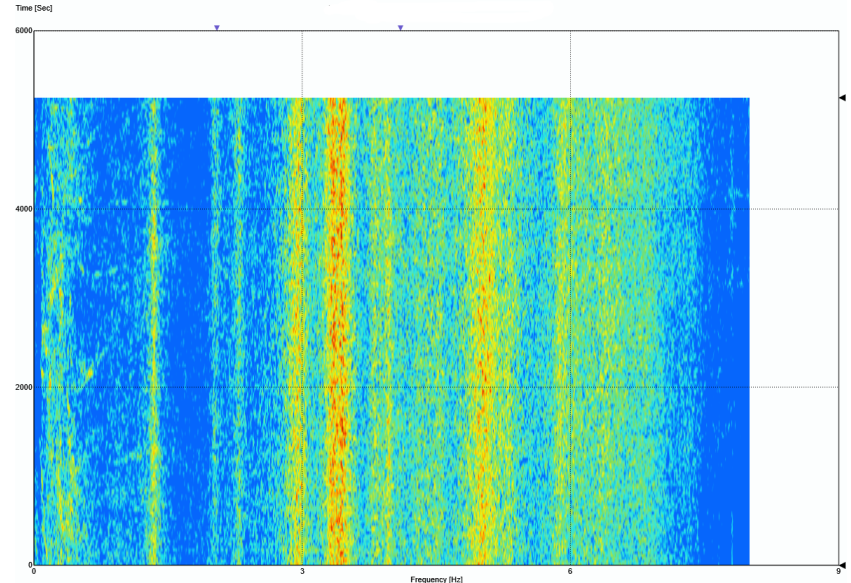

Figure 12: STFT channel 5-9 after the harmonic reduction. Harmonics at $2.05 \mathrm{~Hz}, 4.1 \mathrm{~Hz}, 6.15 \mathrm{~Hz}$ and $7.8 \mathrm{~Hz}$ removed.

Comparing the Figures 11 and 12 it is clear that the three solid red lines representing the response of the ferry towards the harmonic excitation, visible on Figure 11, are removed on Figure 12. No information regarding the modal properties of the ferry without the influence of the harmonic inputs is available hence instead of the tabular comparison of modal parameters a stabilization diagram with corresponding natural frequencies estimated from the data is presented on Figure 13.

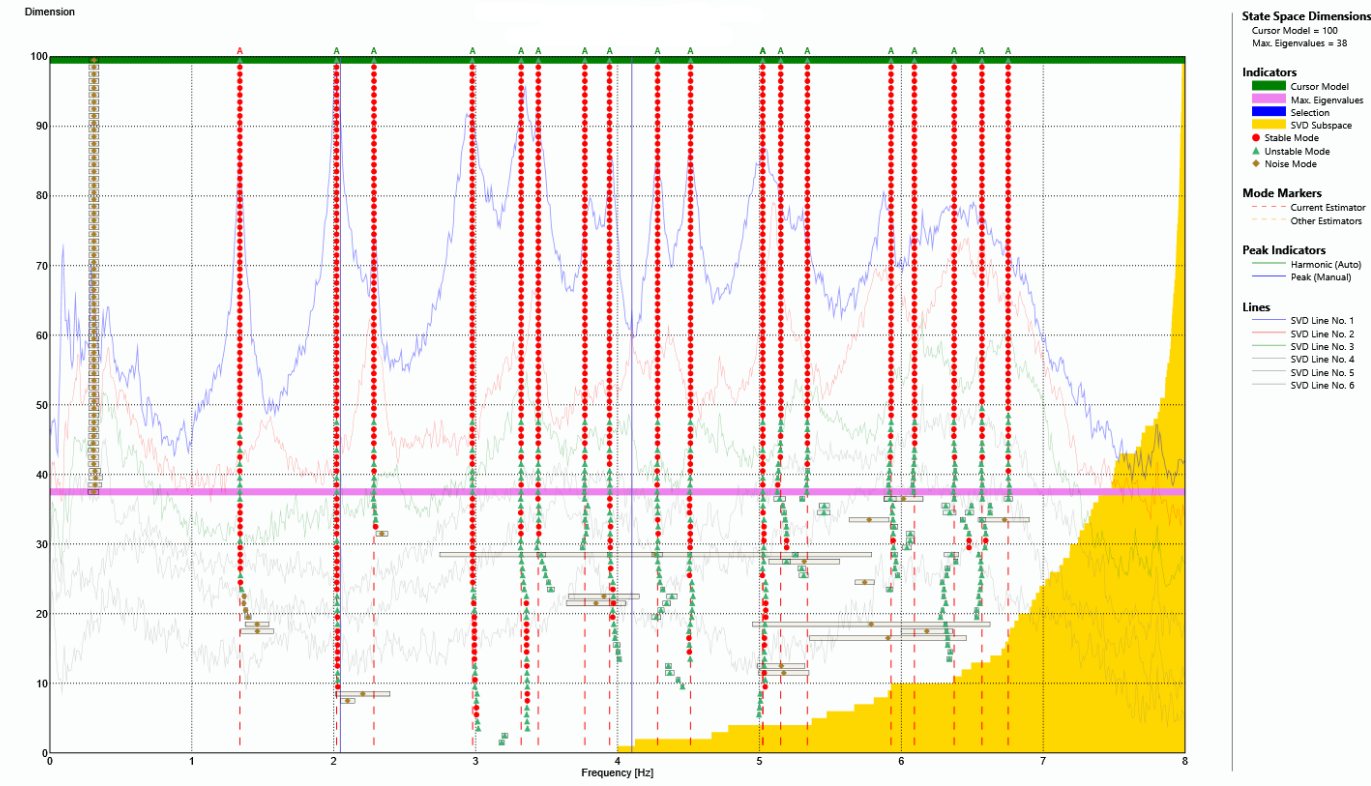

Figure 13: Stabilization diagram of the estimated state space models from the signals after the harmonic removal. SSI-UPCX.

Multiple stable modes are depicted on Figure 13. The harmonic modes previously present in the measurements are not detected 
nor they influence the identification algorithm after the harmonic reduction.

\section{CONCLUSION}

In this paper we presented both the theory and the application of the orthogonal projection-based harmonic reduction method. Proposed algorithm successfully removed the harmonics from the experimental response signals albeit introducing a bias varying in range $1.42-9.92 \%$ between the different modal damping ratios. The source of the error can be explained by a non-linear damping behavior of the plate while excited with strong periodic signals, thus larger than from the random vibration test or a suboptimal choice of a the state space model order for predicting the harmonic realization of the response.

Similar results for the harmonic removal were achieved in context of a real-life application. Developed method removed the harmonic frequencies propagated from the engine of an operating ferry and enabled efficient OMA of the modified response signals.

The application of the method can be extended not only to harmonic reduction \removal but also to removing any arbitrary mode of the system. That might be useful to remove high energy modes present, for instance, in forced excitation during structural testing.

The bottleneck of the method is the choice of the state space model order such that it solely predicts the modal response that corresponds to selected harmonic frequencies. That, along with conducting more tests on a real measurements with strong non-stationary harmonic excitations and large number of channels will be the subject of our future work.

\section{ACKNOWLEDGMENTS}

The authors would like to thank Niels-Jørgen Jacobsen from B\&K Nærum in Denmark for conducting the experimental tests and for sharing its results.

\section{REFERENCES}

[1] Benveniste, A. and Mevel, L., Nonstationary Consistency of Subspace Methods, IEEE Transactions on Automatic Control, Vol. 52, No. 6, pp. 974-984, 2007.

[2] Basseville, M., Benveniste, A., Goursat, M., Hermans, L., Mevel, L. and Van der Auweraer, H., Output-Only SubspaceBased Structural Identification: From Theory to Industrial Testing Practice, Journal of Dynamic Systems, Measurement, and Control, Vol. 123, No. 4, pp. 668-676, 2001.

[3] Balmés, E., Basseville, M., Mevel, L., Nasser, H. and Zhou, W., Statistical model-based damage localization: A combined subspace-based and substructuring approach, Structural Control and Health Monitoring, Vol. 15, No. 6, pp. 857-875, 2008.

[4] Döhler, M., Mevel, L. and Hille, F., Subspace-based damage detection under changes in the ambient excitation statistics, Mechanical Systems and Signal Processing, Vol. 45, No. 1, pp. 207 - 224, 2014.

[5] Peeters, B., Cornelis, B., Janssens, K. and Van der Auweraer, H., Removing Disturbing Harmonics in Operational Modal Analysis, Proceedings of the International Operational Modal Analysis Conference, 2007.

[6] Combet, F. and Gelman, L., An automated methodology for performing time synchronous averaging of a gearbox signal without speed sensor, Mechanical Systems and Signal Processing, Vol. 21, No. 6, pp. 2590 - 2606, 2007.

[7] Randall, R. and Smith, W., New cepstral techniques for operational modal analysis, Proceedings of the First World Congress on Condition Monitoring, 2017.

[8] Bienert, J., Andersen, P. and Aguirre, R., A harmonic peak reduction technique for operational modal analysis of rotating machinery, Proceedings of the International Operational Modal Analysis Conference, 2015.

[9] Pintelon, R., Peeters, B. and Guillaume, P., Continuous-time operational modal analysis in the presence of harmonic disturbancesThe multivariate case, Mechanical Systems and Signal Processing, Vol. 24, No. 1, pp. 90 - $105,2010$.

[10] Peeters, B. and de Roeck, G., REFERENCE-BASED STOCHASTIC SUBSPACE IDENTIFICATION FOR OUTPUT-ONLY MODAL ANALYSIS, Mechanical Systems and Signal Processing, Vol. 13, No. 6, pp. 855 - 878, 1999. 
[11] van Overschee, P. and de Moor, B., Subspace Identification for Linear Systems, Springer, 1 st edn., 1996.

[12] Aoki, M., State space modeling of time series, Springer-Verlag, 3rd edn., 1990.

[13] Jacobsen, N.-J., Andersen, P. and Brincker, R., Eliminating the Influence of Harmonic Components in Operational Modal Analysis, Conference Proceedings, Society for Experimental Mechanics, United States, 2007.

[14] Hoen, C., Subspace Identification of Modal Coordinate Time Series, Proceedings of the 24th International Modal Analysis Conference (IMAC), United States, 2006.

[15] Structural-Vibration-Solutions, ARTeMIS Modal Pro 5.2, 2017.

[16] Döhler, M., Andersen, P. and Mevel, L., Variance computation of modal parameter estimates from UPC subspace identification, IOMAC - 7th International Operational Modal Analysis Conference, Ingolstadt, Germany, 2017.

[17] Rosenow, S.-E., Uhlenbrock, S. and Schlottmann, G., Parameter Extraction of Ship Structures in Presence of Stochastic and Harmonic Excitations, Proceedings of the International Operational Modal Analysis Conference (IOMAC 2007), Vol. 4, 012007.

[18] Jacobsen, N.-J. and Andersen, P., Operational Modal Analysis on Structures with Rotating Parts, Proceedings of the International Conference on Noise and Vibration Engineering, KU Leuven, 2008. 